\title{
BMJ Open Hospital comparison of stroke care in Sweden: a register-based study
}

\author{
Ingrid Lekander, ${ }^{1}$ Carl Willers, ${ }^{2}$ Elisabeth Ekstrand, ${ }^{3}$ Mia von Euler, ${ }^{4}$ \\ Birgitta Fagervall-Yttling, ${ }^{5}$ Lena Henricson, ${ }^{6}$ Konstantinos Kostulas, ${ }^{7,8}$ Mikael Lilja, ${ }^{9}$ \\ Katharina S Sunnerhagen, ${ }^{10}$ Jörg Teichert, ${ }^{11}$ Hélène Pessah-Rasmussen ${ }^{12}$
}

To cite: Lekander I, Willers C, Ekstrand $\mathrm{E}$, et al. Hospital comparison of stroke care in Sweden: a registerbased study. BMJ Open 2017;7:e015244. doi:10.1136/ bmjopen-2016-015244

- Prepublication history and additional material for this paper are available online. To view these files please visit the journal online (http://dx.doi. org/10.1136/bmjopen-2016015244).

Received 18 November 2016 Revised 27 June 2017 Accepted 31 July 2017
CrossMark

For numbered affiliations see end of article.

Correspondence to Dr Ingrid Lekander; ingrid.lekander@ivbar.com

\begin{abstract}
Background and purpose The objective of this study was to estimate the level of health outcomes and resource use at a hospital level during the first year after a stroke, and to identify any potential differences between hospitals after adjusting for patient characteristics (case mix). Method Data from several registries were linked on individual level: seven regional patient administrative systems, Swedish Stroke Register, Statistics Sweden, National Board of Health and Welfare and Swedish Social Insurance Agency. The study population consisted of 14125 patients presenting with a stroke during 2010. Case-mix adjusted analysis of hospital differences was made on five aspects of health outcomes and resource use, 1 year post-stroke.

Results The results indicated that $26 \%$ of patients had died within a year of their stroke. Among those who survived, almost $5 \%$ had a recurrent stroke and $40 \%$ were left with a disability. On average, the patients had 22 inpatient days and 23 outpatient visits, and 13\% had moved into special housing. There were significant variations between hospitals in levels of health outcomes achieved and resources used after adjusting for case mix. Conclusion Differences in health outcomes and resource use between hospitals were substantial and not entirely explained by differences in patient mix, indicating tendencies of unequal stroke care in Sweden. Healthcare organisation of regions and other structural features could potentially explain parts of the differences identified.
\end{abstract}

\section{INTRODUCTION}

In Sweden, approximately 25000 patients suffer a stroke each year, ${ }^{1}$ either caused by bleeding or ischaemia in the brain. Early acute treatment is vital for survival and minimising brain damage due to stroke. Access to acute therapies has increased in recent years, which has had positive impact on the prognosis of the patients' health. Still, many patients have remaining impairments with life-long consequences on functioning ${ }^{1}$ and healthcare costs. ${ }^{2-4}$ The effects of stroke on morbidity, mortality and costs both for the individual and for society are substantial. ${ }^{1-6}$

Healthcare delivery in Sweden is organised into 21 regions, with each region having the authority to decide how to manage, follow-up

\section{Strengths and limitations of this study}

The study is based on register data with good coverage of the stroke population, minimising the risk of selection bias.

- Only including patients with confirmed stroke diagnosis.

- Combination of several data sources enables a holistic view of stroke care, including case-mix variables, health outcomes and resources.

- The method uses established statistical methods to enable case-mix adjusted comparisons between hospitals.

- Register data always have limitations such as missing data, incomplete data and incorrect registrations.

- Potential bias in registration of patient-reported outcomes if those with more severe effects from their stroke could not answer the questions themselves.

- Data on municipality financed home healthcare, informal care and rehabilitation were not available.

and reimburse healthcare costs. Health equity across different regions may not always be granted. Inequalities in health outcomes and health care delivery between different regions or hospitals that cannot be explained by differences in patient mix would contradict not only Swedish legislation ${ }^{7}$ but also declarations by United Nations, WHO and the European Union (EU). ${ }^{8-10}$

The rising cost of healthcare over the past few decades and persistently increasing healthcare cost projections put constraints on the healthcare sector. These costs are driven by factors such as demographic trends, new technologies and increasing demand, ${ }^{11}$ but also by substantial unwarranted variations in clinical practice. ${ }^{12}$ One framework for healthcare management that has recently gained attention, aiming to address parts of these problems, is value-based healthcare. ${ }^{13}$ By focusing on value, defined as the health outcomes (relevant to the patient) achieved in relation to the costs of achieving those outcomes, the framework aims to ensure 
efficient usage of resources. A supportive theoretical framework is that of performance measurement. This framework aims to monitor, evaluate and communicate the extent to which healthcare systems meet key objectives. Indicators for performance measurement often include structural, process and outcome indicators. ${ }^{14}$ As differences in patient population between regions and hospitals may alter a comparison of performance, case-mix adjustment of differences in patient mix is warranted to enable comparison of processes, resources and health outcomes between different regions or hospitals.

For stroke care, no study has previously combined several aspects of both health outcomes and resource use for performance measurement, nor analysed any potential differences in the healthcare delivered that are not related to differences in patient mix. Knowledge of such differences is important for identifying inequalities, as well as possibilities for increasing the value of healthcare for patients with a stroke.

The objective of this study was to estimate the level of health outcomes and resource use during the first year after stroke at a hospital level, and to analyse any potential differences between hospitals after adjusting for patient characteristics (case-mix adjustment).

\section{METHODS}

\section{Study population and data sources}

The study was a retrospective register-based study. The research database consisted of data from patients suffering from stroke between 2007 and 2012, identified in patient administrative systems (PAS) from seven Swedish regions (Jämtland Härjedalen (RJH), Östergötland (RÖ), Dalarna (LtD), Uppsala (LUL), Skåne (RS), Stockholm (SLL) and Västra Götaland (VGR), representing the participating regions in Sveus, www.sveus.se), and covering approximately $60 \%$ of all registered strokes annually in Sweden. PAS contain information on diagnosis and procedure codes related to all healthcare activities registered within the region. Adult patients ( $>18$ years) with stroke were identified at inpatient admission with International Statistical Classification of Diseases and Related Health Problems-10th revision codes I61* (intracerebral haemorrhage), I63* (cerebral infarction) and I64* (unspecified stroke). All data related to the inpatient stay and outpatient visits during the study period were retrieved. All data on a patient's health outcomes and resource use were allocated to the hospital initially treating the patient (regardless of whether the patient was referred to another hospital at a later stage).

Through the unique personal identification numbers, data for the identified patients were linked to multiple other data sources at an individual level: the Swedish Stroke Register (living conditions and patient reported health outcomes), Statistics Sweden (socioeconomic status and mortality), the National Board of Health and Welfare (municipality services) and the Swedish Social Insurance Agency (sick leave and early retirement). These registries all have national coverage above $95 \%$.
The final study population consisted of 14125 patients experiencing a stroke during 2010, registered both in PAS and the Swedish Stroke Register. Choosing 2010 as index year allowed for 2 years of history (enabling case-mix adjustments) and 2-year follow-up (only 1-year results presented in this paper). Patients residing in a region other than where the acute stroke care was performed were excluded from the analysis to allow for complete follow-up.

\section{Study variables}

Identification of key health outcomes, units of resources and factors influencing outcome (case-mix variables) were based on available literature and clinical expertise of the research group. Variables were selected to enable a broad assessment of stroke consequences, addressing both sides of the value equation, limited by data availability. Only variables with good coverage $(>80 \%$ in general, $>70 \%$ for patient-reported variables) were used. The research group consisted of representatives for the regions, patient organisation, specialist organisations, quality registries and Ivbar Institute (R\&D company).

Selected key variables (key data source in parentheses) are as follow:

\section{Health outcomes}

- 1-year overall survival. (Statistics Sweden)

- 1-year recurrent stroke: identified by new acute stroke diagnosis $\left(\mathrm{I} 61^{*}, \mathrm{I} 63^{*}\right.$ or I64*) registered during inpatient admission at an index clinic $>28$ days following the first stroke (coherent with definition used by the Swedish Stroke Register). (PAS)

- Good functioning, defined as the modified Rankin Scale (mRS) 0-2 at 1 year after stroke. The Swedish Stroke Register does not collect mRS but contain variables which allows for estimating $\mathrm{mRS}$ based on the algorithms published by Eriksson et al. ${ }^{15}$ (Swedish Stroke Register)

- Good general health at 1 year after stroke (patient-reported), defined as 'fairly good' or 'very good'. (Swedish Stroke Register)

- 'Full-time work ability'. This was approximated by the proportion of patients not on sick leave or not having taken early retirement at 1 year after stroke, out of the patients $<65$ years who were not on sick leave prior to the stroke. (Swedish Social Insurance Agency)

Resources (first year post-stroke)

- Total inpatient days. (PAS)

- Total outpatient visits in specialist and primary care. (PAS)

- Hours of home care service. (National board of Health and Welfare)

- Proportion of patients moving into special housing for older persons. (National board of Health and Welfare) 
The number of inpatient days and outpatient visits considered as stroke-related were estimated. After review of diagnosis codes, exclusion criteria were applied for specialty care (excluding all healthcare contacts related to tumours and kidney failure). Due to overall poor registration of diagnosis codes in primary care $(<20 \%$ of all contacts), only total number of visits were estimated.

\section{Baseline characteristics}

Case-mix adjustment for relevant patient characteristics at baseline is a prerequisite for meaningful comparisons between hospitals to identify unwarranted variations and inequalities in healthcare. Factors reflecting organisation (e.g. type of hospital, stroke unit and hospital size) and interventions during the acute phase (e.g. reperfusion) were, however, not included in the statistical analysis. While the impact of these factors on outcomes are important, they should not be controlled for when comparing hospital performance; if such differences would be the underlying reason for deviating results, then the comparisons should be meant to unveil these and not to adjust for them. Since the objective was to identify differences in health outcomes and resources not explained by patient mix, only factors that were not a result of the hospitals' care process were included. The selected case-mix variables are as follow:

- Sociodemographic factors:

- Age, sex (PAS)

- Level of education, born outside the EU (Statistics Sweden)

- Single household (Swedish Stroke Register)

- Health profile at baseline:

- Living arrangements, dependence in Activities of Daily Living (ADL) - defined as being dependent on help with dressing and/or going to the toilet (Swedish Stroke Register)

- Prior stroke within last 2 years, total inpatient care (bed-days) the year before stroke, atrial fibrillation and/or hypertension diagnosed within last 2 years (PAS)

- Stroke characteristics:

- Stroke type (PAS)

- Level of consciousness at arrival (Swedish Stroke Register)

Age was categorised into 5-year intervals: $<60 ; 60-64$; $65-69 ; 70-74 ; 75-79 ; 80-84 ; 85-89 ; 89<$ for the statistical analysis. The other categorical variables were used as reported in the registers.

\section{Statistical analysis}

Adjustment of differences in health outcomes and resource use using regression analysis was employed, using a multivariable fixed-effects regression model adjusted for clustering of patients within clinics. Logistic regression was performed for dichotomous outcomes, whereas continuous data were analysed using ordinary least squares (OLS) regression. The full set of predictors was used in all regression models. The case-mix adjusted results indicate the hospital effect on each dependent outcome (referred to as adjusted results).

STATA v.13.0 was used for statistical analyses.

For the overall comparison of hospitals after case-mix adjustment, health outcomes were defined as unexplained better or worse health outcomes if having significant deviations in only one direction for at least three indicators. Exception was made to 'full-time work ability', as this variable concerned a subpopulation and the data used were based on sick leave, which is only a proxy for work ability. This indicator was therefore less robust and not included in the criteria for better health outcomes. For overall assessment of resources, higher or lower level of resources was highlighted in cases where there was a significant deviation of 'inpatient care' and 'move into special housing' in only one direction. Limiting to these two variables was done in accordance with a previous study which demonstrated that these two resource items are the main drivers of the total costs of stroke care. ${ }^{16}$

\section{RESULTS}

The vast majority of the patients had an ischaemic stroke, were conscious on arrival at hospital and were living at home with no home care service prior to the stroke (table 1). Approximately half of the patients were living in single household and the distribution between men and women was even, although women were older on average. Only a small proportion of the patients in this study population received any acute invasive treatment.

There were variations in patient mix between hospitals, both regarding socioeconomic status and health profile at baseline. For example, the proportion of patients born outside the EU varied between $1 \%$ and $14 \%$, mean age ranged from 70 to 80 years, and the proportion of patients with higher education varied between $8 \%$ and 27\%. The proportion of patients who were ADL-dependent varied between $6 \%$ and $20 \%$, and the proportion living in special housing prior to the stroke ranged from $2 \%$ to $22 \%$.

There were also variations in the proportion of patients with haemorrhagic stroke, level of consciousness at arrival and acute invasive treatment given (three hospitals had no acute invasive treatment for their patient population).

\section{Crude rates}

Overall, $73.8 \%$ (95\% CI 73.2 to 74.6 ) of the patients suffering a stroke were still alive 1 year post-stroke, ranging from $66.4 \%$ (95\% CI 62.4 to 70.4 ) to $80.9 \%$ (95\% CI 74.7 to 87.0) on hospital level (see supplementary file 1). Of the deceased patients, more than half died within the first 3 months after their stroke. The proportion of patients with a recurrent stroke (of first-year survivors) was $4.6 \%$ (95\% CI 4.2 to 5.0) with a hospital range of $0 \%-10.1 \%$ (95\% CI 5.4 to 14.8 ).

The proportion of surviving patients with good function (mRS 0-2) amounted to $57.8 \%$ (95\% CI 56.6 to 58.9 ), with a hospital range from $46.9 \%$ (95\% CI 41.9 to 
Table 1 Descriptive statistics of study sample

\begin{tabular}{lllcc}
\hline Category & Variable & Value (total pop) & Min (hospital) & Max (hospital) \\
\hline Demographic and & Age (mean) & 76.32 & 69.99 & 80.63 \\
socioeconomic profile & Male (\%) & 49.37 & 38.84 & 56.02 \\
& College/University degree (\%) & 16.84 & 7.89 & 26.81 \\
& Born outside the EU (\%) & 4.98 & 0.42 & 14.21 \\
& Single household (\%) & 51.92 & 41.20 & 63.16 \\
Health status before stroke & Living in special housing* (\%) & 10.10 & 1.59 & 21.93 \\
& ADL-dependent* (\%) & 11.54 & 5.77 & 19.92 \\
& Prior stroke (-2 years) (\%) & 6.88 & 1.45 & 11.32 \\
Stroke & Inpatient care year -1* (mean days) & 5.33 & 2.84 & 7.34 \\
& Stroke subtype (distribution \%) & & & \\
& Haemorrhagic & 11.68 & 4.38 & 24.40 \\
& Ischaemic & 86.78 & 75.12 & 93.15 \\
& Unspecified & 1.54 & 0.00 & 6.29 \\
\cline { 2 - 4 } Acute invasive treatment & Unconscious at arrival (\%) & 4.98 & 1.79 & 12.75 \\
& Thrombolysis (\%) & 5.84 & 0.00 & 20.00 \\
& Thrombectomy (\%) & 0.83 & 0.00 & 10.22 \\
& Hemicranectomy (\%) & 0.18 & 0.00 & 1.21 \\
\hline
\end{tabular}

*Data on health status before stroke were scarce, therefore proxy variables.

51.8 ) to $77.4 \%$ (95\% CI 61.8 to 93.0 ) 1 year post-stroke. A majority of the patients, $75.2 \%$ (95\% CI 74.2 to 76.2 ) ranging from $67.2 \%$ (95\% CI 58.6 to 75.9 ) to $87 \%$ (95\% CI 78.5 to 95 ), had rated their general health as good.

For surviving patients who worked full-time prior to the stroke, $85.5 \%$ (95\% CI 83.8 to 87.1 ) resumed fulltime work 1 year after stroke (ie, not on sick leave or early retirement).

Use of regional resources amounted to an average of 22.1 inpatient bed-days (95\% CI 21.6 to 22.7), with hospital averages ranging from 13.7 (95\% CI 12.0 to $15.3)$ to 41.1 (95\% CI 34.5 to 47.6 ). The patients had on average 9.4 outpatient visits (95\% CI 9.1 to 9.7) in specialty care, with a hospital range from 1.9 (95\% CI 1.7 to 2.1 ) to 17.4 (95\% CI 14.4 to 20.4). The corresponding number of visits in primary care was 13.7 (95\% CI 13.4 to 14.0) for the total population, with a hospital range from 3.8 (95\% CI 2.5 to 5.2$)$ to 33.8 (95\% CI 27.5 to 40.1 ) visits. In total, the average number of outpatient visits was 23 , with a hospital range of 13-38 visits.

The initial inpatient stay in connection with the acute management of stroke was on average 14 days, that is, $66 \%$ of the total inpatient bed-days during the first year after stroke. Stroke-related resources amounted to approximately 19 inpatient bed-days and 8 outpatient visits within specialty care, that is, the vast majority of the resources were classified as stroke-related.

For municipality financed services, there were substantial differences when comparing patients initially treated at different hospitals. The average home-care service usage amounted to 151 hours. The incremental usage of home-care services the year after experiencing a stroke amounted to 119 (95\% CI 110 to128) with hospital range from 36 hours (95\% CI -4.5 to 77.5 ) to 215 hours (95\% CI 136.6 to 292.6). The proportion of patients moving to special housing the year following the stroke was $13.1 \%$ (95\% CI 12.4 to 13.7), with a hospital average range from $7.3 \%$ (95\% CI 2.9 to 11.7 ) to $18.7 \%$ (95\% CI 9.6 to 27.7 ).

The confidence intervals of crude rates indicated that there were statistically significant differences between hospitals, and several hospitals had significantly lower or higher crude rates than the patient-level average. Variations were also identified between hospitals within respective regions.

\section{Adjusted deviation}

The results showed that there were significant differences in health outcomes between hospitals also after adjustment (figure 1). Six hospitals performed better in terms of health outcomes (marked with light green) and six hospitals performed worse (marked with light red) compared with the other hospitals, with statistically significant deviation on several indicators for health outcomes.

A positive relationship between 1-year survival and recurrent stroke, that is, hospitals with higher survival rates also showing higher rates of recurrent stroke, was identified in 13 hospitals. However, these results were only significant in nine cases (25\% of hospitals). The range in odds ratios for the different health outcomes were larger for 'general health' and 'full-time work ability' than for the other health outcomes analysed.

Regarding resource use, there were significant differences in the adjusted comparison between hospitals (figure 2). The range of inpatient stay spanned from 


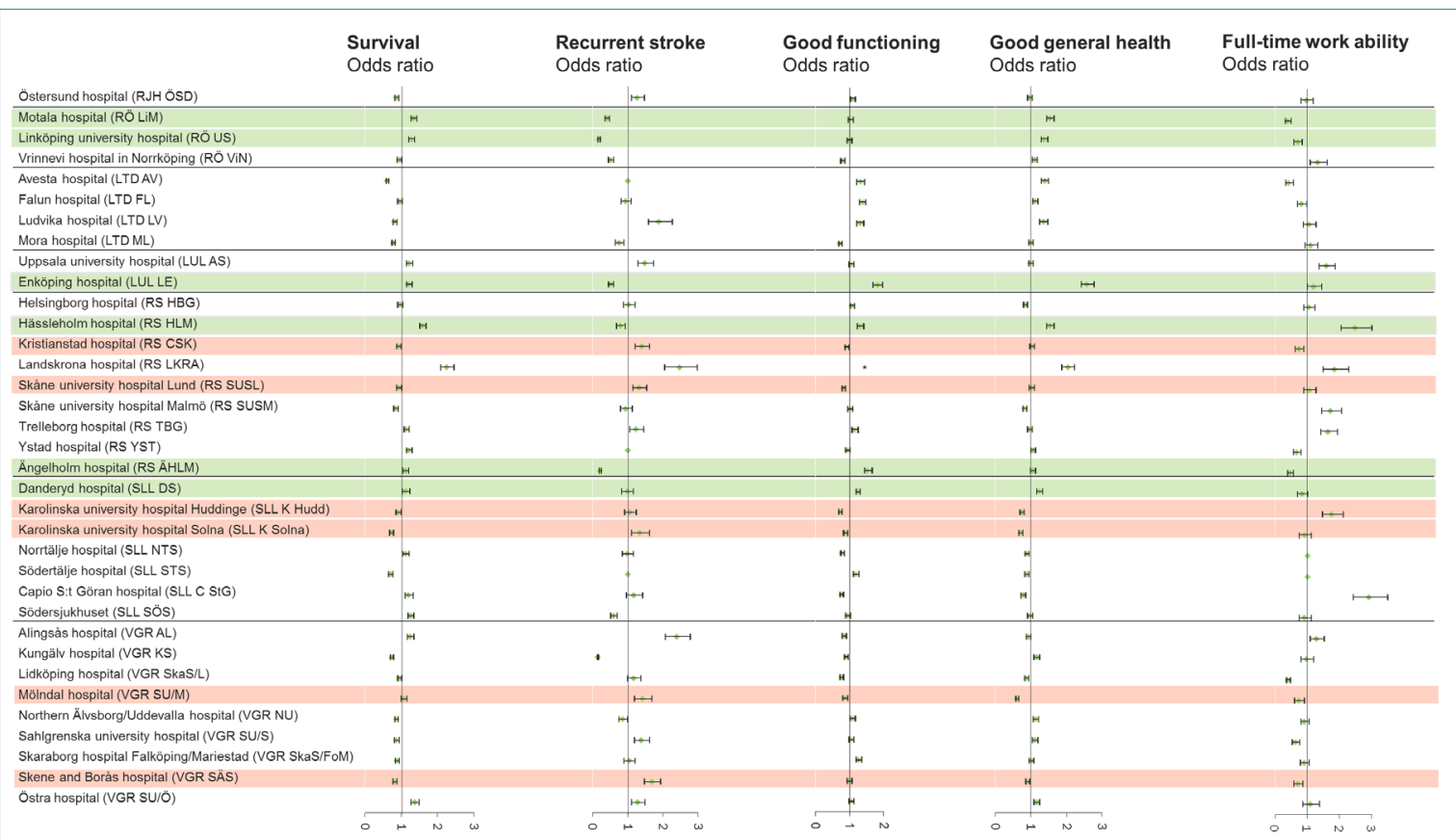

Figure 1 Adjusted deviation in health outcomes 1 year post-stroke. Patients with a stroke in 2010. Colour markers indicate better (green) or worse (red) health outcomes if having significant deviations in only one direction for at least three indicators of outcome. Exception was made to 'full-time work ability', as this variable concerned a subpopulation and the data used were based on sick leave, which is only a proxy for work ability. *Point estimate and $\mathrm{Cl}$ regarding good function for Landskrona hospital excluded for graphical purposes $(\mathrm{OR}=5.54,95 \% \mathrm{Cl} 4.86$ to 6.32 , Landskrona being the hospital with the smallest number of patients).

-8 to +16 days compared with the mean, and the corresponding differences for outpatient visits spanned from -7 to +7 visits for specialty care and -10 to +21 visits for

primary care. There were also substantial deviations for municipality care.



Figure 2 Adjusted deviation in resource use 1 year post-stroke. Patients with a stroke in 2010. Colour markers indicate higher (red) or lower (green) level of resources, highlighted in cases where there was a significant deviation of 'inpatient care' and 'move into special housing' in only one direction. 
Most hospitals showed a pattern of higher levels of resources of one kind (eg, inpatient care) and lower levels of another (eg, outpatient care or special housing). However, five hospitals had lower levels of resource use (marked with light green) and five hospitals had the opposite result, higher levels of resource use compared with the other hospitals (marked with light red). Within regions, trends could be identified with regards to the division of outpatient visits between speciality and primary care e.g. lower number of primary care visits while higher levels of specialist care as in RS, and the opposite relationship in VGR.

\section{DISCUSSION}

\section{Health outcomes and resource use}

In order to improve health equity and increase the value of healthcare, it is important to first identify possible inequalities in healthcare. Sweden has favourable conditions for such a study due to the quality and availability of national registries. Based on such data, the results of this study demonstrated statistically significant variations in health outcomes, not explained by the observed differences in patient mix and thus indicating inequalities on hospital level in Swedish stroke care delivery. Variation in inpatient stay, ${ }^{17}$ costs and survival ${ }^{18}$ between regions and European countries have also recently been identified in another study, confirming the findings. Previous published Swedish data by the National Board of Health and Welfare ('open comparisons') also support this (covering both stroke and other diseases), although these national reports did not consider differences in patient mix. Previous studies assessing resource use and health outcomes in other disease areas have also indicated the importance of measuring these indicators to understand the burden and impact of different chronic diseases, see examples in osteoporosis, rheumatoid arthritis, diabetes and chronic heart failure. ${ }^{19-23}$ Learnings from this study could be used for future research in other countries or disease areas to assess inequalities and value of healthcare, in order to help increasing the value of healthcare delivery. For example, increasing value could be achieved by improving efficiency through introduction of new technologies or treatment processes. ${ }^{24-29}$

This study has confirmed results from other studies with regards to the impact of stroke on health outcomes ${ }^{130} 31$ and resource use following a stroke. ${ }^{17}$ However, there is a discrepancy in the point estimates compared with previous research. The proportion of deceased patients and patients with recurrent stroke was lower, whereas more patients had remaining disability in this study than in previous Swedish studies. ${ }^{31}$ Previous estimates of inpatient stay have also been higher than the results of this study. ${ }^{432}$ These discrepancies can be explained by improvements of acute stroke management over time, which also has been noted in a US-based study. ${ }^{33}$

No conclusions can be drawn as to which resource type provided the best health outcomes, and no clear relationship between resources and health outcomes was detected in line with previous findings. ${ }^{18}$ For example, hospitals in Östergötland and Uppsala showed overall favourable and comparable health outcomes after adjustment. In Östergötland, the patients consumed significantly less outpatient care (specialist/primary combined) although more inpatient care, whereas the opposite was identified in Uppsala. For a complete assessment of the overall resources used, they need to be translated into costs and summarised on hospital level, enabling comparison in a common unit of measurement.

Overall, six hospitals performed better with regards to health outcomes and two hospitals had paired this with significantly lower resource use, implying higher value (Ängelholm hospital, RS, and Danderyd hospital, SLL). Karolinska University Hospital in Solna and in Huddinge (SLL), and Mölndal hospital (VGR) performed worse with regards to health outcomes paired with higher resource use levels, implying a lower value of healthcare. Karolinska Solna also had a patient population deviating from the other hospitals: being the youngest group with highest education, highest proportion of unconscious patients and patients with haemorrhagic stroke. All the aforementioned factors were adjusted for in the analyses, although there might still be factors related to the patient selection affecting the results that could not be controlled for (e.g. National Institutes of Health Stroke Scale (NIHSS)). Furthermore, patients referred to highly specialised stroke care at university hospitals in the larger regions and doing well are re-referred to the local hospital they came from (usually within the same region) while those not doing well remain at the highly specialised unit. In contrast, specialised hospitals in smaller regions have higher proportion of severe stroke cases from other regions (who were excluded from analysis) and fewer hospitals for rereferral within their own region.

The results demonstrated that there were differences between the Swedish regions. Identified variations may stem from different organisation of healthcare on regional level, regional guidelines and/or local traditions and routines (e.g. prehospital care and acute management accessibility). As the accessibility and use of acute invasive treatment in stroke care have increased since 2010, it would be of interest to follow-up on more recent data to investigate if these results have changed. Geographical position and population density of the different regions may also have an effect on the results. Previous studies have assessed the impact of hospital size ${ }^{1834}$ and university hospitals on outcomes, ${ }^{35}$ indicating that there are relationships between these factors that need to be accounted for. This may, for example, explain parts of the results for Östergötland and Uppsala, which both are mid-sized regions with one university hospital each and few other hospitals for acute care of stroke. Future research in healthcare management should investigate the impact of structure and processes on the value of care delivered. 


\section{Strengths and limitations}

A major strength of this study is its base on register data with good coverage of the stroke population, minimising the risk of selection bias. First, the regions' PAS cover all patients with acute stroke diagnosed and registered within each region and all their healthcare contacts. The included regions also covered the majority of the Swedish population. Still, generalisations with regards to other regions and other countries (also subject to other healthcare systems) should be made with caution. Second, the study population consisted of patients with stroke diagnosis registered in both PAS and the Swedish Stroke Register (coverage rate $>95 \%^{1}$ ), implying that only patients with confirmed stroke diagnosis were included.

Another strength is the study's combination of several data sources, enabling analyses to include numerous relevant case-mix variables, health outcomes and resource dimensions over the first year post-stroke. Also, the study population consisted of 14125 patients, providing a solid base for statistical analyses and decreasing the uncertainty of the results.

A register-based study is, however, associated with certain limitations that need to be considered when interpreting the results, such as missing data, incomplete data and incorrect registration. For example, stroke severity data (NIHSS) had too low coverage in the Swedish Stroke Register to be included as case-mix variable, although shown to be important for prediction. ${ }^{36}$ Level of consciousness, which has been shown to be a strong predictor of outcomes ${ }^{37}$ and also a good approximation of the full NIHSS in predictions, ${ }^{38}$ was however included whereby this limitation should have minor impact on the overall conclusions. Additionally, the quality of registrations for healthcare contacts (eg, primary care diagnosis) differed between hospitals, which is why total number of visits were studied (not only stroke-related) in order to minimise the impact of these registration differences. The greater part of the healthcare resource use was nevertheless found to be stroke-related.

Health outcomes were retrieved from several sources and consist of a mixture of hard endpoints and patient-reported outcome measures, dependent on participation of patients and their ability to answer the questions. For some patients with a stroke, these questions were completed by their next of kin, possibly giving other results than if the patient had answered themselves. These patients were most likely severely impaired after the stroke, which may give rise to systematic errors. It is however unlikely that such errors differ between hospitals, and impact on the conclusions are expected to be minor.

The resource dimensions in this paper were chosen to cover all resources, independent of payer. One of the general challenges in stroke management is the diversity in healthcare payers (state, regions and municipalities). Unfortunately, data on municipality financed home healthcare (performed by medical staff), informal care and rehabilitation given were not available and thus these could not be assessed.

\section{CONCLUSIONS}

This study has demonstrated a method of comparing health outcomes and resource use between hospitals while adjusting for differences in patient mix. Such comparisons give valuable information on which hospitals provide the highest value, and if there are any potential inequalities. These results could help foster sharing best practices, also between regions, which can be enhanced when comparisons are performed and transparently communicated on a continuous basis. This will be enabled in an ongoing effort based on the analyses of this study (www. sveus.se). Future research should investigate the impact of different treatment processes on health outcomes to increase the knowledge of best practices.

Differences in health outcomes and resource use between hospitals for patients who experienced a stroke were substantial and not entirely explained by differences in patient mix, indicating tendencies of geographically unequal stroke care in Sweden. Healthcare organisation of regions and other structural features could potentially explain parts of the differences identified. These factors are possible to control on hospital or regional level, whereby improvements are possible which would most likely increase health outcomes for patients who experience a stroke in Sweden and decrease inequalities between patients treated by different hospitals and in different regions.

\section{Author affiliations}

${ }^{1}$ Ivbar Institute AB and Medical Management Center, LIME, Karolinska Institutet, Stockholm, Sweden

${ }^{2}$ Ivbar Institute AB and Department of Clinical Science and Education,

Södersjukhuset, Karolinska Institutet, Stockholm, Sweden

${ }^{3}$ Department of Health Sciences, Lund University, Lund, Sweden

${ }^{4}$ Department of Clinical Science and Education, Södersjukhuset, Karolinska Institutet and Karolinska Institutet Stroke research Network at Södersjukhuset, Stockholm, Sweden

${ }^{5}$ Sweden Occupational Unions, Luleå, Sweden

${ }^{6}$ Swedish Association of Speech and Language Pathologists, Stockholm, Sweden ${ }^{7}$ Department of Neurology, Huddinge Unit, Karolinska University Hospital, Stockholm, Sweden

${ }^{8}$ Department of Clinical Neuroscience, Neuro-Angiological Research Center, Karolinska Institutet, Stockholm, Sweden

${ }^{9}$ Department of Public Health and Clinical Medicine, Family Medicine, Östersund, Umeå University, Östersund, Sweden

${ }^{10}$ Institute of Neuroscience and Physiology, Rehabilitation medicine, University of Gothenburg, Gothenburg, Sweden

${ }^{11}$ Department of Medicine, Landstinget Dalarna, Mora lasarett, Mora, Sweden ${ }^{12}$ Department of Neurology and Rehabilitation Medicine, Skåne University Hospital, Lund, Sweden

Acknowledgements The authors gratefully acknowledge the contributions of: The Swedish Stroke Register, Participating regions, Lena Eriksson, Birgitta Stegmayr, Christina Wikman-Lundbom and Anna Zucco. Partial funding from Forte programme grant 2012/1688 ('Value and choice') is gratefully acknowledged.

Contributors CW and LI: performed the analysis and drafting of manuscript.

Funding Funding for this research was provided by Sveus, through the Ministry of Health and Social Affairs (Dnr S2012/8356/FS) and seven Swedish regional authorities. Sveus is a research collaboration where seven Swedish regions develop systems for value-based monitoring of healthcare. The overarching aim of Sveus is to stimulate value-driven, efficient and patient-centred healthcare. The work has been carried out for a number of different patient groups, of which stroke is one. 
Competing interests IL and CW are employed by lvbar Institute, a research company specialised in healthcare governance and analysis of healthcare data. IL also holds stock in Ivbar Institute. Remaining coauthors did not declare any conflicts of interest.

\section{Patient consent Obtained.}

Ethics approval The study was approved by the Regional Ethical Review Board at Karolinska Institutet in Stockholm (2013/1541-31/5).

Provenance and peer review Not commissioned; externally peer reviewed.

Data sharing statement Patient-level data may not be shared, as patient data regulations in Sweden and regulations of data holders do not allow for this.

Open Access This is an Open Access article distributed in accordance with the Creative Commons Attribution Non Commercial (CC BY-NC 4.0) license, which permits others to distribute, remix, adapt, build upon this work non-commercially, and license their derivative works on different terms, provided the original work is properly cited and the use is non-commercial. See: http://creativecommons.org/ licenses/by-nc/4.0/

(c) Article author(s) (or their employer(s) unless otherwise stated in the text of the article) 2017. All rights reserved. No commercial use is permitted unless otherwise expressly granted.

\section{REFERENCES}

1. Riksstroke Årsrapport 2012,2013 www.riksstroke.se.

2. Gustavsson A, Svensson M, Jacobi F, et al. Cost of disorders of the brain in Europe 2010. Eur Neuropsychopharmacol 2011;21:718-79.

3. Olesen J, Gustavsson A, Svensson M, et al. The economic cost of brain disorders in Europe. Eur J Neurol 2012;19:155-62.

4. Ghatnekar O, Persson U, Glader EL, et al. Cost of stroke in Sweden: an incidence estimate. Int J Technol Assess Health Care 2004;20:375-80.

5. Jennum P, Iversen HK, Ibsen R, et al. Cost of stroke: a controlled national study evaluating societal effects on patients and their partners. BMC Health Serv Res 2015;15:466.

6. Johnston SC, Mendis S, Mathers CD. Global variation in stroke burden and mortality: estimates from monitoring, surveillance, and modelling. Lancet Neurol 2009;8:345-54.

7. Government S. Swedish health and medical services act (Hälso- och sjukvårdslagen, HSL 1982:763), 1982.

8. WHO. Closing the gap in a generation: health equity through action on the social determinants of health: Final Report of the Commission on Social Determinants of Health, 2008.

9. Health inequalities in the EU - Final report of a consortium. Consortium lead: Sir Michael Marmot: European Union, 2013.

10. The United Nations. Universal declaration of human rights.

11. Przywara B. Projecting future health care expenditure at European level: drivers, methodology and results, in Economic Papers 417, European Economy: European Commission, 2010.

12. Peiró S, Maynard A. Variations in health care delivery within the European Union. Eur J Public Health 2015;25 Suppl 1:1-2.

13. Porter ME. What is value in health care? N Engl J Med 2010;363:2477-81.

14. academies I. Performance measurement: accelerating improvement: The National Academies Press, 2006.

15. Eriksson M, Appelros $P$, Norrving $B$, et al. Assessment of functiona outcome in a national quality register for acute stroke: can simple self-reported items be transformed into the modified Rankin Scale? Stroke 2007;38:1384-6.

16. Lekander I, Willers $\mathrm{C}$, von Euler M, et al. Relationship between functional disability and costs one and two years post stroke. PLOS One 2017;12:e0174861.

17. Peltola M, Seppälä TT, Malmivaara A, et al. Individual and regionallevel factors contributing to variation in length of stay after cerebral infarction in six european countries. Health Econ 2015;24 Suppl 2:38-52.
18. Häkkinen $\mathrm{U}$, Rosenqvist $\mathrm{G}$, Iversen $\mathrm{T}$, et al. Outcome, use of resources and their relationship in the treatment of $\mathrm{AMI}$, stroke and Hip fracture at European hospitals. Health Econ 2015;24 Suppl 2:116-39.

19. Ringborg A, Nieuwlaat R, Lindgren $\mathrm{P}$, et al. Costs of atrial fibrillation in five European countries: results from the Euro heart survey on atrial fibrillation. Europace 2008;10:403-11.

20. Ringborg A, Martinell M, Stålhammar J, et al. Resource use and costs of type 2 diabetes in Sweden - estimates from populationbased register data. Int J Clin Pract 2008;62:708-16.

21. Farré $\mathrm{N}$, Vela $\mathrm{E}$, Clèries $\mathrm{M}$, et al. Medical resource use and expenditure in patients with chronic heart failure: a population-based analysis of 88195 patients. Eur J Heart Fail 2016;18:1132-40.

22. Kobelt G, Lindgren P, Geborek P. Costs and outcomes for patients with rheumatoid arthritis treated with biological drugs in Sweden: a model based on registry data. Scand J Rheumatol 2009;38:409-18.

23. Borgström F, Zethraeus $\mathrm{N}$, Johnell $\mathrm{O}$, et al. Costs and quality of life associated with osteoporosis-related fractures in Sweden. Osteoporos Int 2006;17:637-50.

24. Sureshkumar K, Murthy G, Natarajan S, et al. Evaluation of the feasibility and acceptability of the 'Care for Stroke' intervention in India, a smartphone-enabled, carer-supported, educational intervention for management of disability following stroke. BMJ Open 2016:6:e009243.

25. Steen Carlsson K, Andsberg G, Petersson J, et al. Longterm cost-effectiveness of thrombectomy for acute ischaemic stroke in real life: an analysis based on data from the Swedish stroke register (Riksstroke). International Journal of Stroke 2017:174749301770115-13.

26. Ifejika NL, Noser EA, Grotta JC, et al. Swipe out stroke: feasibility and efficacy of using a smart-phone based mobile application to improve compliance with weight loss in obese minority stroke patients and their carers. Int J Stroke 2016;11:593-603.

27. Zhang MW, Yeo LL, Ho RC. Harnessing smartphone technologies for stroke care, rehabilitation and beyond. BMJ Innov 2015;1:145-50.

28. Thorsén AM, Widén Holmqvist L, von Koch L. Early supported discharge and continued rehabilitation at home after stroke: 5-year follow-up of resource use. J Stroke Cerebrovasc Dis 2006;15:139-43.

29. von Koch L, de Pedro-Cuesta J, Kostulas V, et al. Randomized controlled trial of rehabilitation at home after stroke: one-year followup of patient outcome, resource use and cost. Cerebrovasc Dis 2001;12:131-8.

30. Andersen SD, Gorst-Rasmussen A, Lip GY, et al. Recurrent stroke: the value of the CHA2DS2VASc score and the essen stroke risk score in a nationwide stroke cohort. Stroke 2015;46:2491-7.

31. Appelros P, Nydevik I, Viitanen M. Poor outcome after first-ever stroke: predictors for death, dependency, and recurrent stroke within the first year. Stroke 2003;34:122-6.

32. Spieler JF, Lanoë JL, Amarenco P. Costs of stroke care according to handicap levels and stroke subtypes. Cerebrovasc Dis 2004;17:134-42.

33. Stepanova M, Venkatesan C, Altaweel L, et al. Recent trends in inpatient mortality and resource utilization for patients with stroke in the United States: 2005-2009. J Stroke Cerebrovasc Dis 2013;22:491-9.

34. Tsugawa $\mathrm{Y}$, Kumamaru $\mathrm{H}$, Yasunaga $\mathrm{H}$, et al. The association of hospital volume with mortality and costs of care for stroke in Japan. Med Care 2013;51:782-8.

35. Ayanian JZ, Weissman JS. Teaching hospitals and quality of care: a review of the literature. Milbank Q 2002;80:569-93

36. Rost NS, Bottle A, Lee JM, et al. Stroke severity is a crucial predictor of outcome: an international prospective validation study. J Am Heart Assoc 2016:5:e002433.

37. Appelros $P$, Terént $A$. Characteristics of the national institute of health stroke scale: results from a population-based stroke cohort at baseline and after one year. Cerebrovasc Dis 2004;17:21-7.

38. Bray BD, Campbell J, Cloud GC, et al. Derivation and external validation of a case mix model for the standardized reporting of 30day stroke mortality rates. Stroke 2014;45:3374-80. 